\title{
Congestion Aware Link Cost Routing For MANETS
}

\author{
Tripti Sharma $^{1}$ and Dr. Vivek Kumar ${ }^{2}$ \\ ${ }^{1}$ IPEC, Ghaziabad, UP, India and ${ }^{2} \mathrm{GKV}$, Haridwar, UK, India.
}

\begin{abstract}
Due to the dynamic topology, self-configuration and decentralized nature of Mobile Ad hoc Network (MANET), it provides many benefits in wireless networks and is easy to deploy. But the transmission of data over ad hoc networks has elevated many technical issues for successful routing. Congestion is one of the important issues which cause performance degradation of a network, due to long delay and high packet loss. This paper proposes a Congestion aware Link Cost Routing for MANET where the protocol finds a path with optimized linked cost based on SNR, Link delay, and the and remaining battery power. Along with this optimization, in this protocol, every node finds its congestion status and participates in the route discovery on the basis of its status. Data forwarding is also done based on the congestion status at the time of forwarding. The protocol results in better performance in terms of packet delivery fraction, end to end delay, throughput, and packet drop when compared to existing protocols.
\end{abstract}

\section{KEYWORDS}

Self-configuration, Packet loss, Congestion, Link cost

\section{INTRODUCTION}

In MANET, due to the mobility of hosts, the network topology of ad hoc network may change rapidly and unpredictably over time. Several advantages have been offered by Ad hoc Networks including appropriate service coverage, simple network maintenance, and low cost [1]. Apart from these advantages MANET also has some limitations like limited transmission capacity and buffer at the nodes and the multi-hop relay intercommunications. Due to limitation of resources, congestion can occur in the route, during the data transmission from source to the destination. It will lead to greater packet loss, long delay and waste of resource utilization. The autonomous nodes in an ad hoc network may suffer from congestion, since the buffer size of the node is limited. When the traffic received at a node is greater than the capacity of its buffer, congestion occurs at that node. So, one of the vital task that an ad hoc network must perform is to resolve the congestion problem. One of the ways to resolve congestion is to limit the delay and buffer overflow caused by network congestion and deliver improved performance of the network.

There are various QoS parameters through which the network resources can be utilized in a better way. In [2], the QoS has been provided through the cost of the link which is calculated with three parameters: Signal to Noise ratio, link delay and the remaining node energy. In this paper, the work proposed in [2] is extended by incorporating congestion avoidance as an additional parameter of QoS. The proposed work is experimented in two different scenarios to show the improved results as compared to the traditional AODV and the link cost based improvement of AODV i.e. LCAODV as detailed in [2]. The comparison is performed for four metrics: packet delivery fraction, end to end delay, throughput and packet drop. 
International Journal of Computer Networks \& Communications (IJCNC) Vol.8, No.3, May 2016

The remaining parts of this paper are organized as follows: section 2 describes the related work. In section 3 the detail of the proposed protocol is given. The performance study is explained with the results of two experiments in section 4 . The conclusion of the paper is provided in section 5.

\section{RELATED WORK}

In [3], a dynamic load-aware routing protocol (DLAR) is proposed which considers the intermediate node routing loads as the primary route selection metric. The protocol also observes the congestion status of active routes and reconstructs the route when the nodes of the route have their interface queue overloaded. The authors in [4] found that AODV is unsuccessful under traumatic network traffic situations. So, they proposed a modified AODV protocol which adds the nodes with little queuing delays into the route to the destination. The modification called as CADV (Congestion Aware Distance Vector), improves the quality of the route, but CADV does not adapt to congestion. No solution has been given to deal with the problem when an existing route becomes deeply congested.

The problem of congestion becomes more visible especially in large-scale transmission of high traffic such as multimedia data, where congestion is more probable and the negative impact of packet loss on the service quality is more of significance. The authors in [5] argued that routing should be aware of and adaptive to congestion. They proposed a unicast routing protocol which tries to minimize congestion at the place where it has been occurred and the protocol adapts to it should the congestion occur during the network lifetime. This problem of congestion actually emerges at MAC or network layer, so an approach to deal with this problem is to control it on these layers only. An extremely high network load is a crucial issue associated with medium access and packet forwarding. Congestion is a foremost reason for packet drops in ad hoc networks. In Mobile Ad hoc Network the uncontrolled congestion can lead to a congestion collapse of the network and the throughput of the network can severely deteriorate [6].

In [7], L. Chen et al. proposed the cooperative design of congestion control, routing and scheduling for ad hoc wireless networks. The rate constraint and scheduling constraints are formulated with multi commodity flow variables, and fixed wireless channels are formulated with resource allocation in the networks. This dual algorithm is extended to deal the networks with the time varying channels and adaptive multi-rate devices. The authors then generalize the results to a queuing network model aided by a set of mutually dependent parallel servers with time-varying service skills. Many communication networks problem can be modeled with this design.

The main objective of congestion control is to make the network perform better by minimizing the delay and buffer overflow produced by network congestion. Congestion control is implemented at the transport layer in wired networks and is normally designed distinctly from the functions of other layers [8]. To improve the performance of TCP in multi-hop wireless networks $\mathrm{X}$. Wang et al. [9], proposed a cross layer hop by hop congestion control scheme which manages the congestion response across the MAC, network, and transport layer protocols. This scheme attempts to define the real cause of a packet loss and then directs the suitable congestion control response among the MAC, network, and transport layer protocols.

In [10], authors proposed a technique called EDAODV (Early Detection congestion and control routing), to detect the congestion before the failure of route and find another non-congested path. As more number of packets are being transmitted through a network, congestion occur in the network that results in retransmission of lost packets, high packet loss rate, loss of energy and bandwidth, and re-routing uncertainty. Authors in [11], describe that delays and packet losses need not necessarily be caused by network congestion, but these can be misinterpreted as congestion losses. 
The authors in [12] proposed an early congestion detection and adaptive routing in MANET called as EDAPR. It finds a route to a destination by constructing an NHN (non-congested neighbors) neighbors list. All the nodes involved in primary path periodically calculate status of their queue. With this technique, the congestion that is likely to happen at a node can be detected early by sending warning message to NHN nodes. The ancestor NHN node can find an alternate path to a destination immediately by applying adaptive path mechanism when get aware of this situation. Therefore, EDAPR improves the performance of the network.

In [13], authors proposed a mobile agent based congestion control technique using AODV routing protocol to evade congestion in ad hoc network. In this technique the routing information and nodes congestion status is carried by some mobile agents in ad-hoc network. When mobile agent moves over the network, it can select a neighbor node which has less- burden, as its next hop and the node's congestion status is updated in the routing table. The nodes can get the dynamic network topology in time with the support of mobile agents.

Authors consider certain TTL value and varying threshold value in [14], to establish the long route connection and also measure the length of the queue variably so that if buffer size of the node is occupied then no packet drop occurs from the queue; it means the queue size varies according to data. This improves the performance of AODV protocol with minimized packet loss. A dynamic estimation of congestion with controlled routing has been proposed in [15], which uses the average length of the queue to estimate the present level of congestion of a node and communicate this level to its neighbors by sending a message. Then the neighbors provide their effort to find a non-congested alternate path towards the destination. This mechanism ensures a reliable transmission of data in the mobile ad hoc networks.

\section{Congestion Aware Link Cost Routing}

We propose a Congestion aware Link Cost Ad hoc On demand Distance Vector (CLCAODV) protocol that finds a path with optimized link cost between the source and the destination. Along with this optimization every node estimates its congestion status based on estimated average queue size when either it initiates the route discovery or it receives a packet (either Route request (RREQ) or data). It then (1) Transmits the packet when congestion status is non-congested as indicated by the comparison of average queue size with its minimum value, (2) Drops the packet with a calculated probability when the average queue size is within a range (as specified by the minimum and maximum threshold values), and (3) Drops the packet if average queue size is beyond the maximum threshold value of the average queue size.

The link cost of the optimum route is discovered on the basis of three parameters: Signal to Noise Ratio (SNR), Link delay and the Node lifetime. The signal to noise ratio (SNR) is used to examine the effect of noise on unpredictable wireless communication network. It is defined as the ratio of signal power to the noise power and is calculated at the receiving node. Link delay is the time period between the packets sent by the sending node and the received at the receiving node. The link delay is calculated at the node after reception of every RREQ packet by using its packet creation time and its time of reception. The Node lifetime is also an important parameter for route selection and is provided by an estimated value of remaining battery lifetime in each RREQ packet.

In the optimal path the delay is expected to be minimized while the Node Lifetime and SNR values are desired to be maximized. Therefore, in the calculation of composite metric (ie. Link Cost) the three parameters (SNR, Node lifetime and delay) are normalized. For selecting a route each QoS parameter requires a weight factor $\left(\mathrm{K}_{1}, \mathrm{~K}_{2}\right.$ and $\mathrm{K}_{3}$, respectively) to calculate the link cost at node $\mathrm{j}$ as in equation (1). 
$\operatorname{LinkCost}_{j}=\left(K_{l} * \operatorname{SNR}_{i, j}\right)+\left(K_{2} * \operatorname{Delay}_{i, j}\right)+\left(K_{3} * N L T_{j}\right)$

where $K_{1}+K_{2}+K_{3}=1$

$\mathrm{SNR}_{i, j}$ is the SNR of the link from node $\mathrm{i}$ to node $\mathrm{j}, \mathrm{Delay}_{i, j}$ is the delay of link from node $\mathrm{i}$ to node $\mathrm{j}$ and $N L T_{j}$ is the Node Life Time of node $\mathrm{j}$. For the efficient path selection a max-min approach is used here, in which the link cost of the path is the minimum of the costs of all the links of the path and for routing, the source node chooses the path with the maximum value of the cost of all the link costs value of all the paths.

Based upon the actions performed by the node according to its congestion status, packets may be dropped by the node and thus the current path may fail. In this situation, the route discovery will be performed again to find an optimal congestion aware path. Thus, the main aim of CLCAODV is to find a congestion-aware optimized route between the source and the destination. This protocol consists of the following components:

i. Congestion Status Estimation

ii. LCAODV route discovery

\subsection{CONGESTION Status Estimation [16]}

The algorithm estimates the congestion status of the node with an average size of queue. The average size of queue $\left(\mathrm{Q}_{\mathrm{avg}}\right)$ is calculated with the previous average queue size and the size of instantaneous queue $\left(\mathrm{Q}_{\text {inst }}\right)$ modified by queue weight parameter as in equation (2).

$\mathrm{Q}_{\mathrm{avg}}=(1-\mathrm{w}) * \mathrm{Q}_{\mathrm{avg}}+\mathrm{Q}_{\mathrm{inst}} * \mathrm{w}$

where $\mathrm{w}$ is a constant parameter representing the queue weight. Since $\mathrm{w}$ is a constant parameter, a short-term increase in queue size because of bursty traffic or transient congestion need not result in a significant increase in the average queue size. The size of the transient burst that the queue can accommodate without dropping packets provides the setting of the value of $\mathrm{w}$. Therefore, the $\mathrm{Q}_{\text {avg }}$ changes much slower than the $\mathrm{Q}_{\text {inst. }}$. The value of $\mathrm{w}$ has been taken as 0.002 , because of the justification given in [17]. The minimum and maximum threshold values for the queue size (Qsize) are: $\mathrm{Th}_{\min }=5, \mathrm{Th}_{\max }=15$. The optimal setting for minimum threshold value depends on the demand of the communication that either low average delay is desired or high link utilization is desired. The higher minimum threshold value would allow the burstier traffic at the node to achieve average link utilization. The link speed, propagation delay and maximum buffer size also put impact on the minimum threshold value.

The queue is non-congested and will not drop the packet if the $\mathrm{Q}_{\text {avg }}$ is less than $T \mathrm{~h}_{\min }$. If $\mathrm{Q}_{\text {avg }}$ is greater than $\mathrm{Th}_{\max }$ then the queue is congested and will drop every arriving packet. If the $\mathrm{Q}_{\text {avg }}$ is greater than $\mathrm{Th}_{\min }$ and less than $\mathrm{Th}_{\max }$, then the current packet will be dropped with probability $\mathrm{p}$ which can be calculated as in equations (3) and (4).

$\mathrm{p}_{\mathrm{d}}=0.1\left(\mathrm{Q}_{\mathrm{avg}}-\mathrm{Th}_{\min }\right) /\left(\mathrm{Th}_{\max }-\mathrm{Th}_{\min }\right)$

$\mathrm{p}=\mathrm{p}_{\mathrm{d}} /\left(1-\mathrm{C}^{*} \mathrm{p}_{\mathrm{d}}\right)$

where $\mathrm{p}_{\mathrm{d}}$ (packet dropping probability) varies linearly between 0 and 0.1 , and $\mathrm{C}$ gives the count of the packet since the last dropped packet. The objective of average queue length is to integrate all the traffic variations, and it follows the long-term changes of instantaneous queue which reflects the insistent congestion in the network. Figure 1 shows the algorithm for congestion status estimation 


\subsection{LCAODV ROUTE DISCOVERY}

The route discovery process starts if a non-congested node has to send some data to any other node. The source node broadcasts RREQ packet to its neighbors. Each receiving node will calculate the link cost with SNR and delay of the link and its own remaining lifetime, store it and will broadcast RREQ further. When the destination receives the request packet it calculates the link cost and put it in the reply packet to send towards the source. When an intermediate node will receive the reply it will compare the stored link cost and the link cost received in the reply packet and update the reply packet with the minimum link cost value. These replies arrived at the source have the lowest available link cost of the corresponding path. The source node starts the data transmission with the first received reply. When the next reply packet arrives at the source, it compares the link cost of the new path with the link cost of current path and chooses the path with the maximum link cost for data transmission and ignores the other paths. Figure 2 shows the algorithm for LCAODV route discovery.

\section{Performance Study}

The proposed protocol CLCAODV is implemented with network simulator NS2 [18, 19], and the results are compared with LCAODV and AODV protocols.

\subsection{Performance Metrics}

We considered the following important metrics in this evaluation:

I. Packet delivery fraction (PDF): The ratio between the number of packets received by the destination and the number of packets sent by the source.

II. End-to-end delay (E2E): The delay a packet suffers from leaving the sender to arriving at the receiver.

III. Throughput: Total data received by receiver in a given time interval.

IV. Packet drop: Total number of data packets failed to reach their destination across the network. Packet drop is measured as the number of packet lost with respect to packet sent.

\subsection{Simulation CONFiguration}

The network consists of a $1000 \mathrm{~m} * 1000 \mathrm{~m}$ terrain size with 100 number of nodes. The range of radio signal is $250 \mathrm{~m}$ and the channel bandwidth is $8 \mathrm{Mbps}$. The MAC layer is based on IEEE 802.11 distributed coordination function. The channel propagation model we used is the 2- ray ground reflection model. And the mobility model used is random way point model. The energy model is also needed to find the energy of the node. The MAC layer interface queue can store 50 packets. At the network layer routing buffer can hold up to 500 data packets. The data packets keep waiting in this buffer for which the process of route finding has begun but no reply has been received yet. The routing protocols used for comparison are CLCAODV, LCAODV and AODV. The number of connections for source and destination pair varies from 10 to 50 and data flow used is variable-bit rate (VBR) that varies from 50 packets to 250 packets per second. The maximum speed of a node is $10 \mathrm{~m} / \mathrm{s}$ and the simulation time is $100 \mathrm{~s}$. We performed two experiments for this simulation to observe the behavior of protocols. 
International Journal of Computer Networks \& Communications (IJCNC) Vol.8, No.3, May 2016

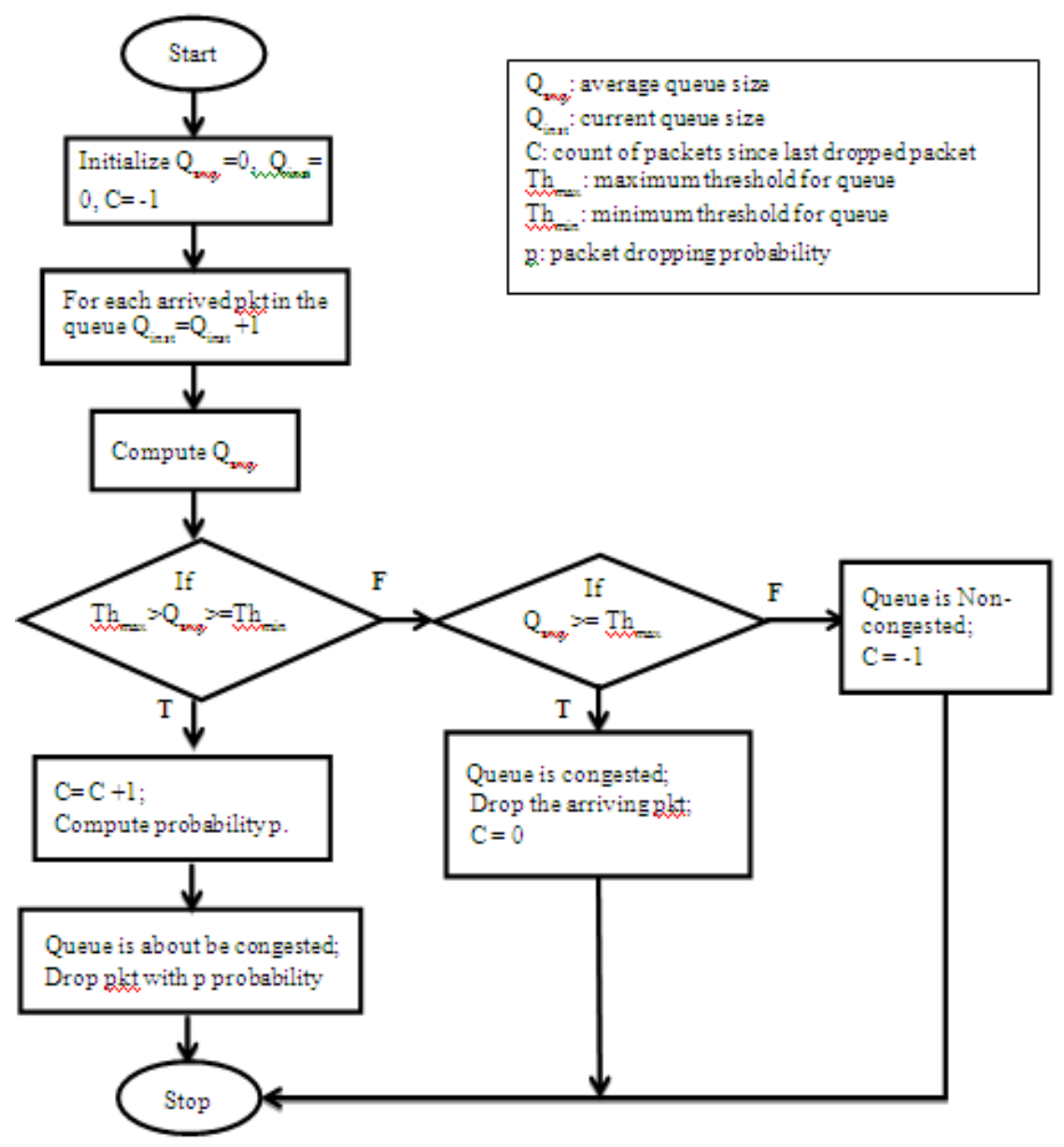

Figure 1. Congestion Status Estimation 


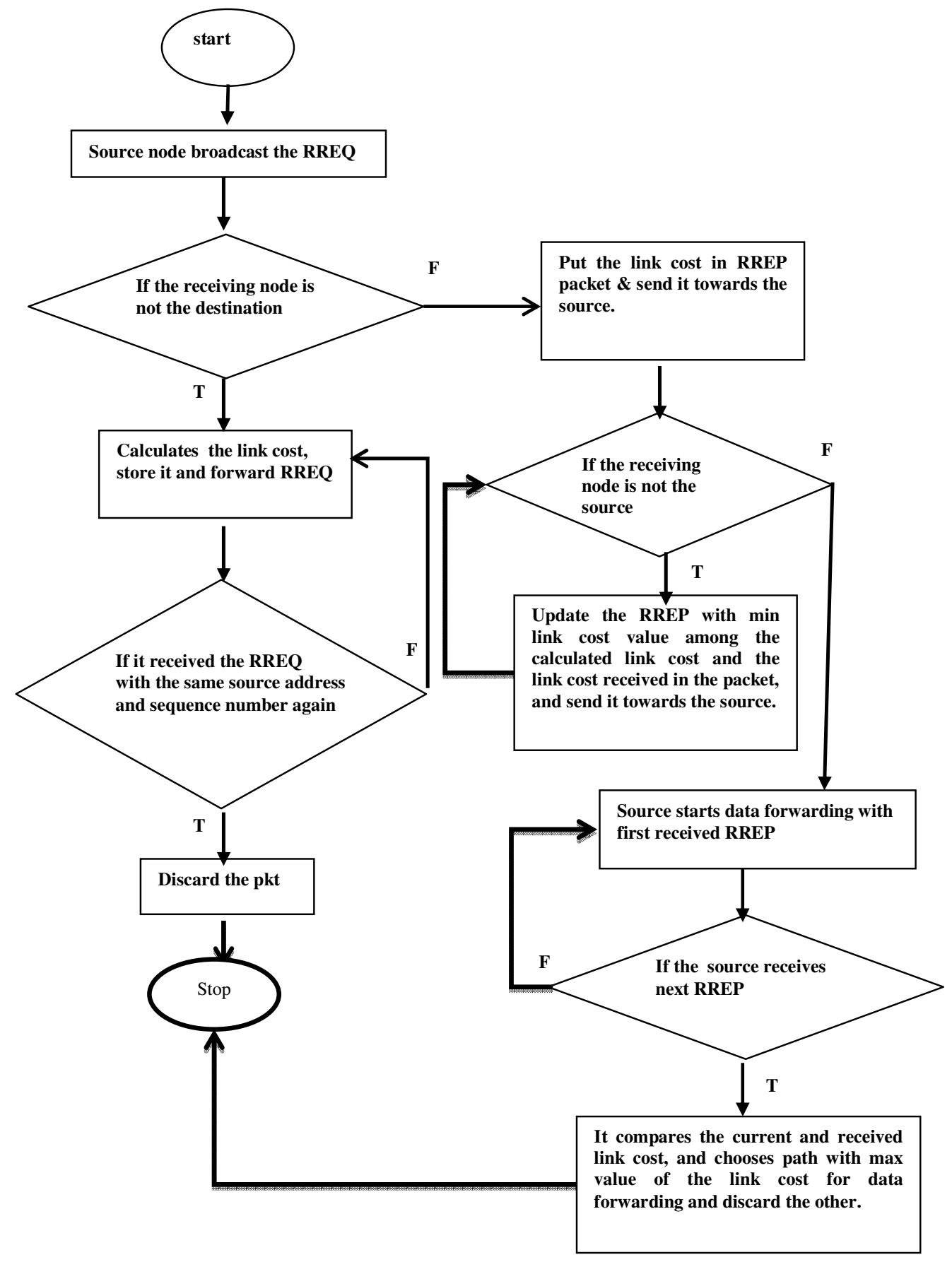

Figure 2. LCAODV route discovery

\subsection{EXPERIMENT 1: VARYing NUMBER OF CONNECTIONS}

In experiment 1 of this simulation, the number of connections (source and destination) varies from 10 to 50, Variable Bit Rate (VBR) rate is 50 packets per second, maximum node speed is 10 $\mathrm{m} / \mathrm{s}$. The results of this experiment are shown in Figure 3(a-d). 
Figure 3(a) shows the packet delivery fraction for the three protocols with the increasing connections. The packet delivery fraction decreases as the number of connections increase. When the number of connections is less, the number of nodes commencing the route discovery procedure is also less so the packet delivery fraction is high. When the number of flows increases up to 50, more RREQ packets are produced and transferred; this leads to a more ingestion of queue at the node, triggering network congestion. This situation leads to lesser number of data packets being conveyed at the destinations, thus degrading the performance of network. As shown in the figure the packet delivery fraction of CLCAODV is high because it chooses noncongested nodes in the path. The packet delivery fraction for varying number of connections of CLCAODV is increased by an average of $4 \%$ when compared to the AODV. Whereas when compared to the LCAODV, it increases by an average of $2.2 \%$. The difference in the attained packet delivery fraction is because of the reduction of the queue occupancy at the node. As a result, more communication bandwidth is available for data transmission for successful delivery.

The end-to-end delay of CLCAODV shown in Figure 3(b) is low while transmitting data packets to the destinations. The result shows 14\% reduction in end to end delay than AODV and 10\% reduction in end to end delay than LCAODV for varying number of connections. When the number of flows increases, the network carries more traffic. The AODV and LCAODV acquired congestion due to increasing traffic flow but the CLCAODV is not affected to the same degree by increasing traffic because it selected the congestion free path by directing the traffic through noncongested nodes only.

The results of throughput for the three protocols are shown in Figure 3(c). Throughput of the network decreases as the number of connections increase. When the number of connections is less, more data is received by the receiver so the throughput of the network is high. When the number of flows increases up to 50, more packets are being transmitted by the nodes per unit time; this leads to high queue occupation at the node, causing the situation of network congestion. This leads to less number of packets received at the receiver, thus degrading the performance of network. As shown in the figure, the throughput of CLCAODV is high in comparison of AODV and LCAODV since CLCAODV considers the congestion avoidance mechanism. The throughput for varying number of connections of CLCAODV is increased by an average of $10 \%$ when compared to AODV. Whereas, when compared to the LCAODV, it increases by an average of $5.8 \%$ over the network.

We can infer from Figure 3(d) that the packets dropped for varying number of connection is less for CLCAODV when compared to AODV and LCAODV. Since the route selected in CLCAODV is the optimum non- congested route and the reduction in packet drop is achieved by monitoring the status of queue at the node, and hence preventing unnecessary routing re-discovery when the network suffers from congestion due to increasing number of flows. The results show an average of $4.4 \%$ less drop of packets for varying number of connections as compared to AODV protocol and $2.9 \%$ less than LCAODV protocol. 
International Journal of Computer Networks \& Communications (IJCNC) Vol.8, No.3, May 2016

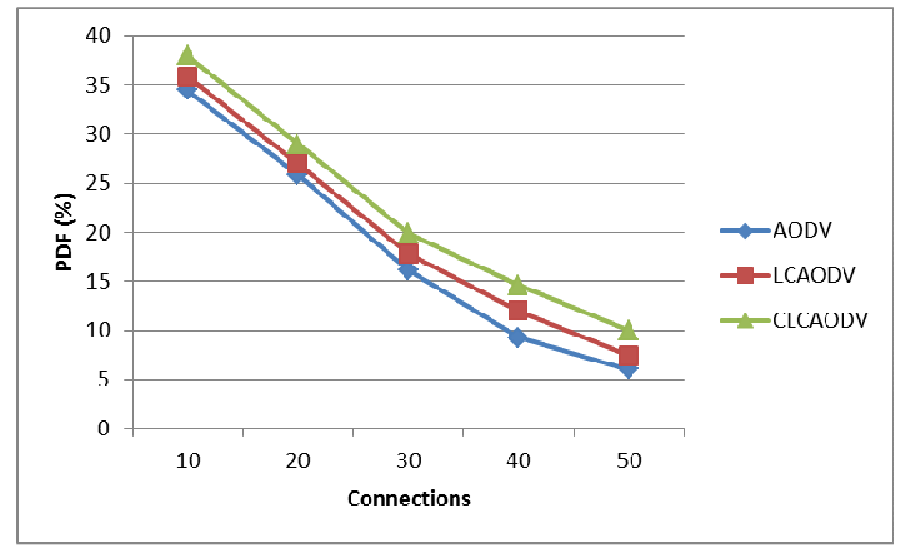

Figure 3(a). Packet delivery fraction vs Connections

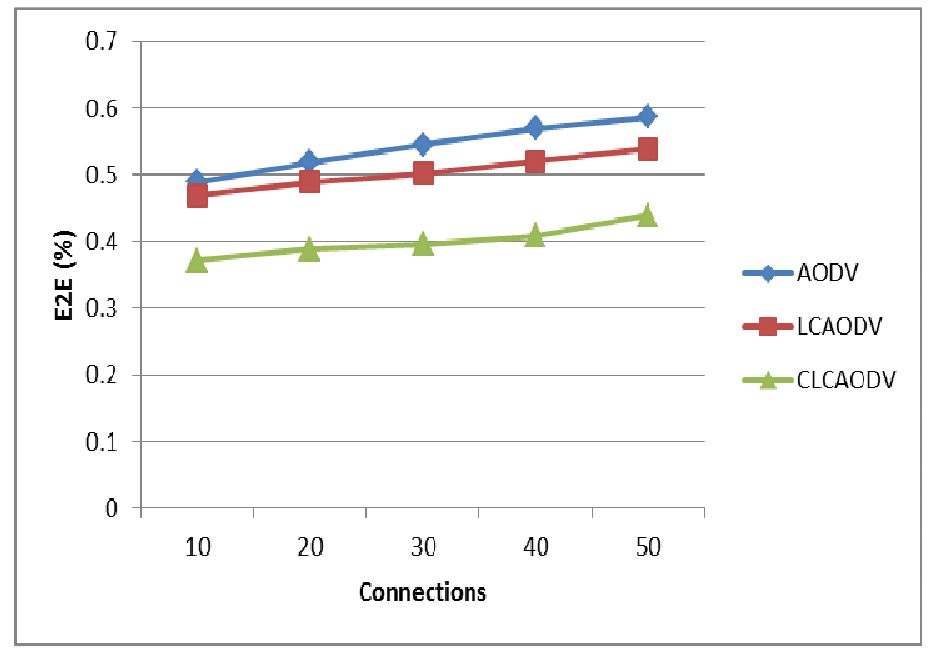

Figure 3(b). End-to-end delay vs connections

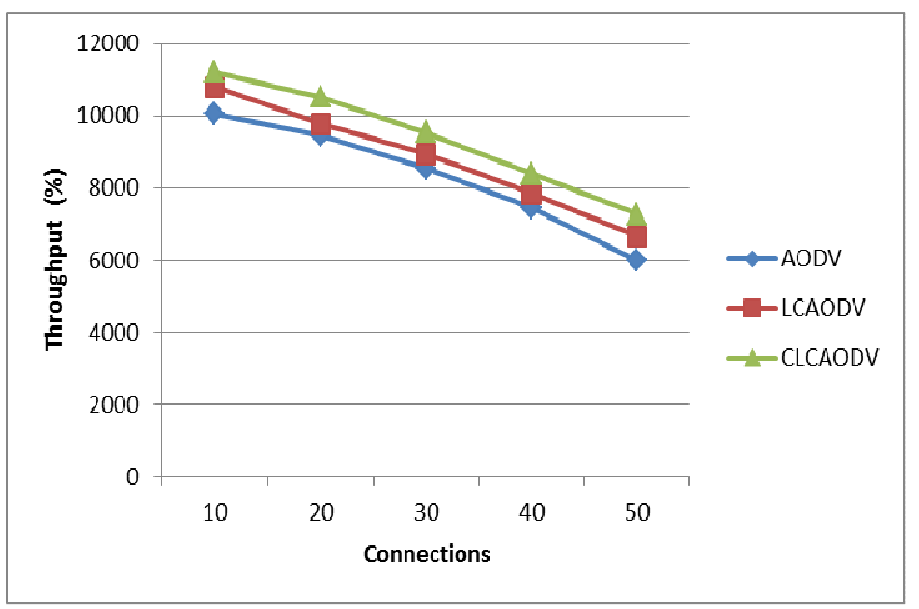

Figure 3(c). Throughput vs connections 


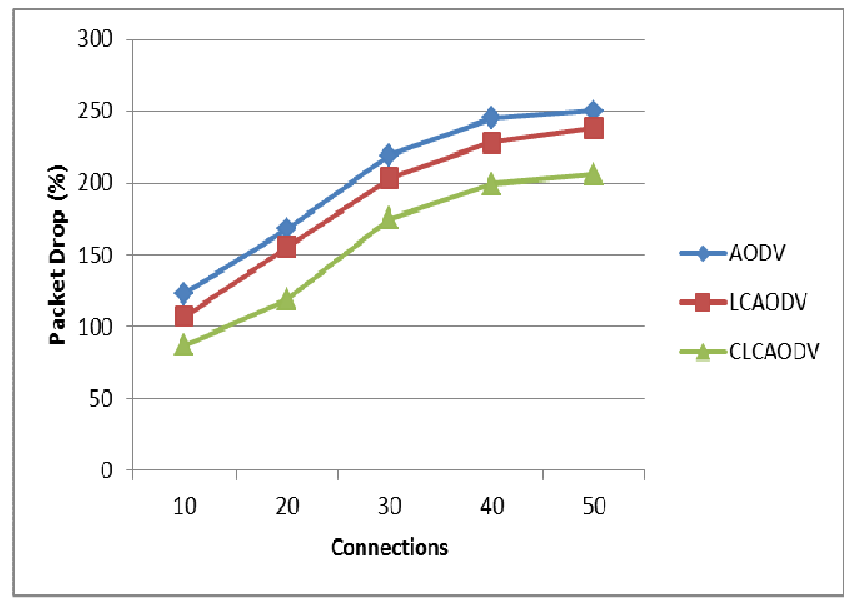

Figure 3(d). Packet drop vs connections

\subsection{EXPERIMENT 2: VARYING VBR LOAD}

In experiment 2 of this simulation, the number of connections (for different sources and destinations) is kept at 20. The VBR rate varies from 50 packets per second to 250 packets per second. The results of this experiment are shown in figure 4(a-d).

In Figure 4(a) when the packet rate is small (up to 50 packets per second), the three protocols CLCAODV, LCAODV and AODV deliver nearly same number of packets because the traffic in the network is not yet hefty. But when the packet rate become high (up to 250 packets per second), the network becomes congested and so the packet delivery fraction becomes reduced. The CLCAODV protocol deals with this situation through the use of congestion avoidance using congestion status estimation technique in the data forwarding. The CLCAODV shows an average improvement of $7.5 \%$ in the packet delivery ratio for varying packet rate than the AODV, and $4 \%$ improvement than the LCAODV.

For the varying packet rate the end-to-end delay experienced by all protocols is shown in Figure 4(b). When the packet rate increases the network is engaged with more number of packets and the end to end delay increases due to the increased waiting time for the packets in the queue. The CLCAODV demonstrates a reduction in delay over the LCAODV and AODV by applying a mechanism (based upon the controlled average queue size) that keeps the average queue size low. The CLCAODV gives an average reduction of end to end delay for varying packet rate by $14 \%$ as compared to AODV and a reduction of $12 \%$ than LCAODV protocol.

In Figure 4(c), the three protocols CLCAODV, LCAODV and AODV give high throughput because the traffic in the network is not yet hefty when the packet rate is small (50 packets per second). But when the packet rate becomes high (up to 250 packets per second), the network becomes occupied with high traffic and so the queue at the node becomes congested which may cause packet drop and thus reduced throughput. The CLCAODV protocol deals with this situation through the use of mechanism that keeps throughput high by making the average queue size adaptable to the nature of the traffic. The CLCAODV shows an average improvement of $4 \%$ in the throughput for varying packet rate than the AODV, and $2.3 \%$ improvement than the LCAODV. 
International Journal of Computer Networks \& Communications (IJCNC) Vol.8, No.3, May 2016

The number of dropped packets is increased with increased packet rates as shown in Figure 4(d). For the low packet rate the difference in the number of dropped packets is not much. But when the packet rate is high the network become congested. In this situation, the difference in the number of dropped packets is remarkable, since the CLCAODV can deal with the congestion easily. The result shows an average decrement of $6.5 \%$ and $3.6 \%$ in the packets drop for varying packet rate when compared to AODV and LCAODV respectively.

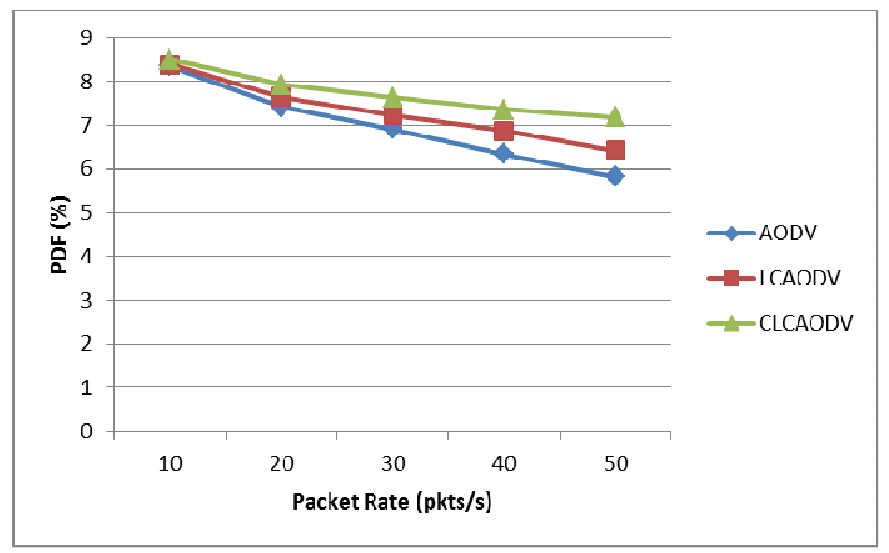

Figure 4(a). Packet delivery fraction vs packet rate

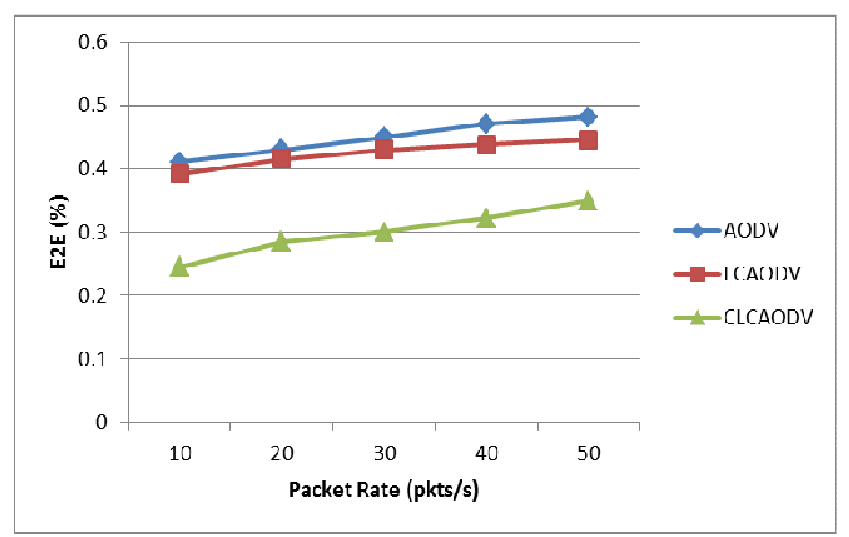

Figure 4(b). End-to-end delay vs packet rate

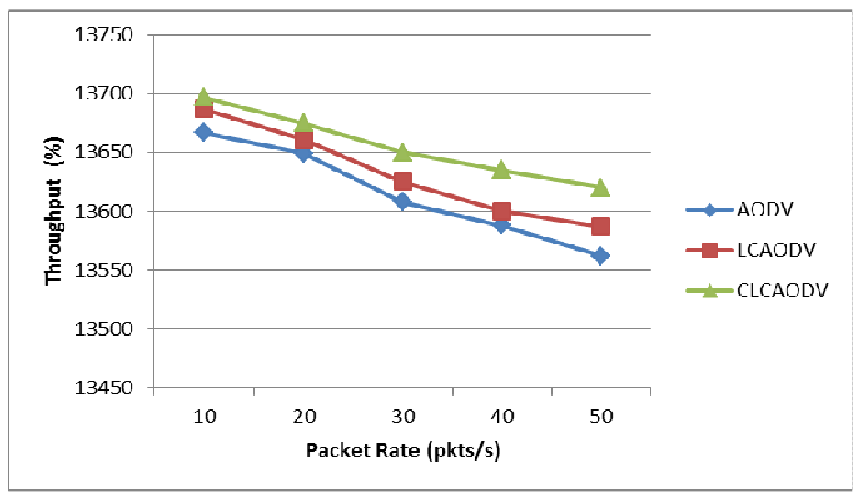

Figure 4(c). Throughput vs packet rate 


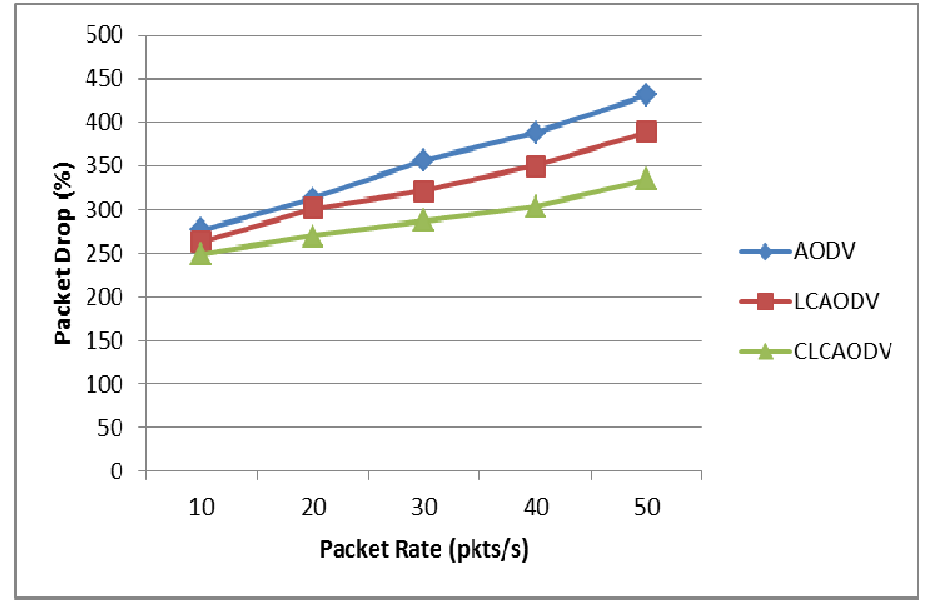

Figure 4(d). Packet drop vs packet rate

\section{CONCLuSiON}

In this paper, a congestion estimation technique has been used as QoS parameters to improve the quality of services for Mobile Ad hoc Networks. We have proposed a protocol that can find the efficient path with optimized link cost and can detect the status of the congestion at the node by analyzing the traffic fluctuation. The CLCAODV algorithm shows considerable performance over AODV and LCAODV.

\section{REFERENCES}

[1] Ramanathan, R. and Redi, J., "A Brief Overview of Ad Hoc Networks Challenges and Directions", IEEE Communications Magazine, Vol. 40, Issue 5, 2002, pp. 20-22.

[2] Sharma, T. and Kumar, V., "Link Cost Routing for MANETs", International Journal of Computer Science \& Engineering Technology, Vol. 7, No. 2, Feb. 2016, pp. 24-32.

[3] Lee S.J., and Gerla M., "Dynamic Load-Aware Routing In Ad Hoc Networks", Proceedings of IEEE International Conference on Communications, 2001, pp. 3206-3210.

[4] Lu Y, Wang W, Zhong Y, Bhargava B. "Study of Distance Vector Routing Protocols For Mobile Ad hoc Networks", Proceedings of IEEE International Conference on Pervasive Computing and Communication (PerCom) 2003, pp. 187-94.

[5] Tran, D.A., Raghavendra, H., "Congestion Adaptive Routing in Mobile Ad Hoc Networks", IEEE Transactions on Parallel Distributive System, Vol. 17, Issue 11, 2006, pp.16-28.

[6] Lochert, C., Scheuermann, B., and Mauve, M., "A Survey On Congestion Control For Mobile AdHoc Networks", Wireless Communication and Mobile Computing, John Wiley \& Sons Ltd., Vol. 7, Issue 5, 2007, pp. 655-76.

[7] Chen, L., Lowy, S.H., Chiangz, M., Doyley, J.C., "Cross Layer Congestion Control Routing And Scheduling Design In Ad Hoc Wireless Networks", IEEE Proceedings Of 25th International Conference On Computer Communication, INFOCOM, 2007, pp. 1-13.

[8] Yingqun, Y., Giannakis, G.B., "Cross-Layer Congestion And Contention Control For Wireless Ad Hoc Networks", IEEE Transactions on Wireless Communications, Vol. 7, Issue 1, 2008, pp. 37-42.

[9] Wang, X., Perkins, D. "Cross-layer Hop-by-hop Congestion Control in Mobile Ad Hoc Networks", Proceedings of IEEE Wireless communication and networking conference, WCNC. 2008, pp.2456 2461.

[10] SenthilKumaran T, Sankaranarayanan V., "Early Detection Congestion and Control Routing in MANET", Proceedings of the Seventh IEEE and IFIP International Conference On Wireless and Optical Communications Networks (WOCN 2010), 2010, pp. 1-5.

[11] Yen, Y-S, Chang, H-C, Chang, R-S, Chao, H-C, "Routing With Adaptive Path and Limited Flooding For Mobile Ad Hoc Networks", Elsevier Transaction on Computers and Electrical Engineering, Vol. 36, Issue 2, 2010, pp.280-290. 
[12] Senthilkumaran, T., Sankaranarayanan, V., "Early Congestion Detection and Adaptive Routing In MANET”, Egyptian Informatics Journal, Vol. 12, Issue 3, 2011, pp. 165-175.

[13] Sharma, V. K. and Bhadauria, S. S., "Mobile Agent Based Congestion Control Using AODV Routing Protocol Technique For Mobile Ad-Hoc Network", International Journal of Wireless \& Mobile Networks (IJWMN), Vol. 4, No. 2, April 2012, pp. 299-314.

[14] Sharma, A. and Singh, A., "Improvement in Routing Behavior of AODV Protocol in MANET". International Journal of Advanced Research in Computer Engineering \& Technology (IJARCET), Vol. 2, Issue 12, December 2013, pp. 3047-3051.

[15] Senthilkumaran, T, Sankaranarayanan, V., "Dynamic Congestion Detection and Control Routing In Ad hoc Networks", Journal of King Saud University - Computer and Information Sciences, Vol. 25, Issue 1, 2013, pp. 25-34

[16] Floyd, S., Jacobson, V., "Random Early Detection Gateways For Congestion Avoidance", IEEE/ACM Transactions on Networking, Vol. 1, Issue 4, 1993, pp. 397-413.

[17] Floyd, S., "RED: Discussion of Setting Parameters". [Online]. Available from: $<$ http://www.icir.org/floyd/REDparameters.txt>.

[18] McCanne, S. and Floyd, S. ns Network Simulator, http: //www. isi.edu/nsnam/ns.

[19] Fall, K. and Varadhan, K. The VINT project. The NS manual.

\section{AUTHORS}

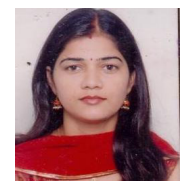

Tripti Sharma has completed her Master of Technology in Computer Science (2009) from Uttar Pradesh Technical University, Lucknow, India and now she is a research scholar of Gurukul Kangri University, Haridwar, India. Her research interest is Quality of Service support for Mobile Ad hoc Networks.

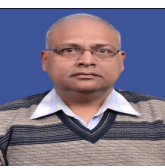

Vivek Kumar has completed his Master degree in Applied Mathematics (1985-1987) and Computer Applications (1987-1988) from University of Roorkee (now IIT), Roorkee , India and Ph.D. from Gurukul Kangri University, Haridwar, India. His research interest includes machine learning, distributed computing and wireless adhoc networks 